\title{
Auf dem Weg in die postfaktische Politik?
}

\author{
Olaf Hoffjann / Lucas Seeber*
}

Die Erfolge von Politikern wie Donald Trump und Boris Johnson sowie von Parteien wie $\operatorname{der} A f D$ haben dazu geführt, dass mit Begriffen wie Desinformation, dem so genannten Bullshit oder Post-Truth Politics bzw. postfaktischer Politik bewusste Verstöße gegen die Wabrheitsnorm bzw. eine wachsende Irrelevanz von Wabrheit in der strategischen politischen Kommunikation intensiv diskutiert werden. Im Mittelpunkt dieses Beitrags steht die erweiterte Perspektive postfaktischer Politik, die in Deutschland empirisch bislang kaum erforscht ist. Für die Existenz einer solchen postfaktischen Politik ist vor allem die Unterstellung derselben relevant. Der reflexive Charakter postfaktischer Politik impliziert, dass man nicht nur selbst postfaktische Politik unterstellt, sondern auch anderen unterstellt, dass sie von einer solchen postfaktischen Politik ausgehen. Dabei kann zwischen zwei Typen unterschieden werden: „Postfaktische Bürgerinnen“ unterstellen Politikerinnen eine weitgehende Gleichgültigkeit gegenüber der Wabrbeit. „Postfaktische Akteurinnen" erachten einen solchen gleichgültigen Umgang mit der Wabrheit für legitim. Diese Aspekte stehen im Mittelpunkt dieses Beitrags: Wie bewerten Politikerinnen, Pressesprecherinnen und Journalistinnen die Verbreitung und Akzeptanz von Lügen und Bullshit in Deutschland? In welchem Ausmaß nehmen sie sich selbst als Teil einer postfaktischen Politik wabr? Und ergänzend: Wie reagieren Journalistinnen auf Politikerinnen, denen sie eine feblende Relevanz der Wabrbeit unterstellen. Dazu wurden in einer Onlinebefragung insgesamt 758 Abgeordnete des Bundestages und aller Landtage, der Mitglieder der Bundespressekonferenz und aller Landespressekonferenzen sowie Pressesprecherinnen von Parteien, Fraktionen und Ministerien auf Bundes- und Landesebene befragt. Die Ergebnisse zeigen u. a., dass etwas mebr als die Hälfte der Befragten sich in einer postfaktischen Demokratie wähnt, während mehr als 90 Prozent der Befragten eine Politik erwarten, die ernsthaft, verbindlich und mit Wabrheitsanspruch auftritt.

Schlüsselwörter: Desinformation, postfaktische Politik, Befragung, politische Kommunikation, Medien-Politik-Verhältnis

\section{Heading toward a Post-factual Politics?}

The success of politicians such as Donald Trump and Boris Johnson, as well as populist parties such as the AfD in Germany, has led to a feverish discussion of terms such as disinformation, 'bullshit' or post-truth politics. There has been an increase in studies examining the willful violation of the truth norm and the decreasing relevance of truth in strategic political communication. This paper focuses on the broader perspective of post-truth politics - a topic that has to date been the subject of barely any empirical research in Germany. The existence of a post-truth politics hinges first and foremost on the assumption of a post-truth politics. The reflexive character of post-truth politics implies that one not only normalizes post-truth politics, it also assumes that others do so as well. Here, a distinction

* Prof. Dr. Olaf Hoffjann, Otto-Friedrich-Universität Bamberg, Institut für Kommunikationswissenschaft, An der Weberei 5, 96045 Bamberg, Deutschland, olaf.hoffjann@uni-bamberg.de, ORCID https://orcid.org/0000-0002-4049-4581.

Lucas Seeber, M. A., Otto-Friedrich-Universität Bamberg, Institut für Kommunikationswissenschaft, An der Weberei 5, 96045 Bamberg, Deutschland, lucas.seeber@uni-bamberg.de, ORCID https://orcid.org/0000-0001-8605-7996.

Die Autoren bedanken sich herzlich bei Jana Keil für ihre Mitarbeit im Ergebnisteil. 
can be made between two types: 'Post-truth citizens' assume that politicians are largely indifferent to the truth. 'Post-truth actors' consider such indifference to the truth to be legitimate. These are the foci of this article: How do politicians, spokespersons, and journalists assess the spread and acceptance of lies and bullshit in Germany? To what extent do they perceive themselves as being part of a posttruth politics? In addition, how do journalists react to politicians who they accuse of being disconnected from the truth? Toward these aims, we surveyed a total of 758 members of the federal and state parliaments, members of the German Federal Press Conference, and all state press conferences as well as spokespersons for parties, parliamentary groups, and ministries at the federal and state levels. The results show, among other things, that slightly more than half of the respondents believe themselves to be living in a post-truth democracy, while more than 90 percent expect politics to be serious, binding, and that it has a claim to truth.

Keywords: disinformation, post-truth politics, survey, political communication, media-politics interactions

\section{Einleitung}

Mit dem Aufkommen von Politikern wie Donald Trump und Parteien wie der AfD haben gewöhnungsbedürftige Schlagworte wie Fake News, der so genannte Bullshit oder Post-Truth eine neue Aufmerksamkeit in öffentlichen und schnell auch in wissenschaftlichen Diskursen erlangt. Dabei kann zwischen einer engen und erweiterten Perspektive postfaktischer Politik differenziert werden (ähnlich Higgins 2016). Während sich z. B. die deutsche Kommunikationswissenschaft vor allem für Begriffe wie Fake News bzw. der Desinformation interessiert, sie definiert (z. B. Quandt et al. 2019; Scholl 2019; Zimmermann \& Kohring 2018) oder den redaktionellen Umgang damit untersucht (z. B. Boberg et al. 2018), wurde im angloamerikanischen Raum zusätzlich die erweiterte Perspektive diskutiert: Welche Folgen ergeben sich für politische, aber auch für andere gesellschaftliche Diskurse, wenn immer häufiger gelogen wird bzw. man gleichgültiger gegenüber der Wahrheit wird, oder dies zumindest unterstellt wird?

Damit rücken vor allem Begriffe wie Post-Truth in den Mittelpunkt, die in einer ersten Annäherung mit Harsin (2018, S. 2) verstanden werden können ,as a social and political condition whereby citizens or audiences and politicians no longer respect truth [...] but simply accept as true what they believe or feel". Eine postfaktische Gesellschaft prägt also grundsätzlich, dass Wahrheit unwichtiger wird. Dies können zum Beispiel Lügen als unaufrichtige Behauptungen sein. Die typischere Form einer Behauptung in einer postfaktischen Gesellschaft ist jedoch der so genannte Bullshit: eine Behauptung, bei der sich der Behauptende gar nicht mehr für die Unterscheidung von wahr vs. unwahr interessiert (Meibauer 2013; Frankfurt 2006).

Für die Existenz einer solchen postfaktischen Politik ist weniger das tatsächliche Ausmaß von Lügen und Bullshit wichtig, sondern vielmehr die Unterstellung derselben (Higgins 2016). Der reflexive Charakter postfaktischer Politik impliziert, dass man nicht nur selbst postfaktische Politik unterstellt, sondern auch anderen unterstellt, dass sie von einer solchen postfaktischen Politik ausgehen. Zudem wird zwischen so genannten „postfaktischen Bürgerinnen“" die Politikerinnen eine weitgehende Gleichgültigkeit gegenüber der Wahrheit unterstellen, und so genannten „postfaktischen Akteurinnen“, die dies für legitim erachten, unterschieden.

Diese Aspekte stehen im Mittelpunkt der Forschungsfragen dieses Beitrags: Wie bewerten Politikerinnen, Pressesprecherinnen und Journalistinnen die Verbreitung und

1 In dem Beitrag werden das generische Femininum und Maskulinum kapitelweise abwechselnd verwendet. 
Akzeptanz von Lügen und Bullshit in Deutschland? Und: Wie hoch ist der Anteil an Politikerinnen, Journalistinnen und Pressesprecherinnen, die sich als Teil einer postfaktischen Politik wabrnebmen? Die Studie untersucht damit einen ausgewählten Aspekt der politischen Kommunikationskultur von Eliten in Politik und Journalismus (Blumler \& Gurevitch 1995; Pfetsch 2003). Dazu wurden in einer Onlinebefragung Abgeordnete des Bundestages und aller Landtage, der Mitglieder der Bundespressekonferenz und aller Landespressekonferenzen sowie Pressesprecherinnen von Parteien, Fraktionen und Ministerien auf Bundes- und Landesebene befragt.

Postfaktische Politik rückt die besondere Verantwortung des Journalismus in den Mittelpunkt. Wo Politikerinnen und ihre Pressesprecherinnen sich nicht länger für die Wahrheit interessieren, könnte der Journalismus mit einer kritischen Berichterstattung zur Restabilisierung der Wahrheitsnorm beitragen. Daher wird sein Umgang mit postfaktischer Politik in der dritten Forschungsfrage näher untersucht. Wie reagieren Journalistinnen auf Politikerinnen, denen sie eine feblende Relevanz der Wabrbeit unterstellen? Thematisieren sie dies explizit als Normverstoß oder seben sie dies als „Teil des Spiels" an?

Damit untersucht die Studie erstmals das Ausmaß einer postfaktischen Kommunikationskultur in Deutschland. Warum hat eine solche erweiterte Perspektive in Deutschland bislang kaum eine Rolle gespielt? Ein Grund hierfür mag sein, dass Deutschland keinen Regierungschef wie Donald Trump, Boris Johnson und Jair Bolsonaro hat, dem vielfach Lügen oder postfaktisches Verhalten unterstellt worden wäre. Außerdem gibt es noch keinen vergleichbaren tiefen Graben zwischen zwei großen politischen Lagern wie in den USA, der einhergeht mit der Polarisierung der Medienlandschaft (Lewandowsky et al. 2017) - trotz der Wahlerfolge einer rechtspopulistischen Partei wie der AfD in Deutschland.

\section{Postfaktische Politik}

Der Begriff Post-Truth mag bereits zu Zeiten des Watergate-Skandals benutzt worden sein (Mair 2017), doch erst seit den Jahren der US-Präsidentschaft von Donald Trump und den Kampagnen der Brexiteers ist er so breit genutzt und diskutiert worden, dass Post-Truth 2016 zum Oxford Dictionaries Wort des Jahres gewählt wurde. Die Zahl der Veröffentlichungen zum Thema Post-Truth bzw. zur postfaktischen Politik als seiner deutschen Übersetzung explodierten in der Folge (Harsin 2018). Auch wenn der Begriff Post-Truth allgemein auf unterschiedliche gesellschaftliche Sphären bezogen werden kann, stand von Beginn an die Politik im Mittelpunkt des Interesses, wie die Vielzahl an Publikationen zu postfaktischer Politik belegen (z. B. Gibson 2018; Hopkin \& Rosamond 2018). Seltener wird das Postfaktische als Problem der Wissenschaft und nur in Ausnahmen wurde es in anderen Feldern strategischer Kommunikationsforschung wie dem Marketing erörtert (z. B. Arora 2020).

Was kennzeichnet postfaktische Politik bzw. Gesellschaften? In den zahlreichen Definitionen lassen sich insbesondere vier Merkmale identifizieren (Hoffjann 2020):

1. Offenkundig ist das erste Merkmal: Wahrheit wird grundsätzlich unwichtiger. Postfaktische Politik prägt der Verzicht auf Evidenz, interne Konsistenz und die Tatsachensuche (Lewandowsky et al. 2017). Die Folge: „the very distinction between factual truth and falsehood has become irrelevant" (Kalpokas 2019, S. 71).

2. Aus der zunehmenden Irrelevanz von Fakten folgt zweitens die Frage, was an ihre Stelle tritt: Dies sind Gefühle oder persönliche Überzeugungen - „feelings are more accurate than facts“ (McIntyre 2018, S. 174): „A defining element of the post-truth 
condition is a certain relativism where personal values, beliefs and emotions take precedence." (Ihlen et al. 2019, S. 2)

3. Während im Kontext von aktueller Desinformation oder Bullshit als Zuschreibung einer Aussage oder eines Kommunikators vor allem die Verantwortung der Kommunikatoren oder der redaktionelle Umgang mit Desinformationen untersucht wird, interessieren sich die Ansätze zur postfaktischen Politik drittens für eine gesellschaftsweite Perspektive, bei der es weniger um einzelne Lügen oder Bullshit von Politikern geht, sondern vielmehr um eine weithin geteilte Annahme, dass es zu einer Entkopplung von Politik und Wahrheit kommt (Haacke 2019, Kap. 2.1.).

4. Und viertens wird postfaktische Politik schließlich in aller Regel als Ergebnis eines Veränderungsprozesses beschrieben (Corner 2017) oder als Beginn einer neuen Ära (d'Ancona 2017). Dies setzt jedoch voraus, dass es früher eine Ära der Wahrheit gegeben haben müsse (Corner 2017; Harsin 2018). Ausführungen zu einer solchen Wahrheitsära finden sich im Diskurs jedoch kaum. Vogelmann (2018, S. 20) vermutet, dass man hier auch nicht fündig würde: „we should doubt the historical claim inherent in the diagnosis of a 'post-truth era', for we will not find an 'era of truth' that came before".

Die Ursachen postfaktischer Politik sind bislang zumeist nur oberflächlich beschrieben worden. Vielfach beschränken sich die Analysen auf einige Hinweise zum Medienwandel und der politischen Kultur, selten wird eine breitere Perspektive wie bei Lewandowsky et al. (2017) eingenommen, die die Ursachen im politischen, technologischen und sozialen Kontext verorten. Es ist naheliegend, dass viele dieser und nachfolgend skizzierten Entwicklungen eng zusammenhängen und sich einander bedingen. Eine gesellschaftstheoretische Perspektive wird dabei zumeist ignoriert. Eine Ausnahme ist Gibson (2018), der in dem Konzept der Reflexivität von Giddens (1995) den zentralen Bezugspunkt von postfaktischer Politik erkennt: „Post-truth politics refers to the specific political and rhetorical strategies that emerge from, and take advantage of, the circular relationship between the endless reflexivity of late modernity and a loss of faith in institutions that anchor truth claims“ (Gibson 2018, S. 3170). Damit hängen eine weitere Individualisierung und Wertepluralisierung zusammen, die zu einer zunehmenden Perspektivenvielfalt führen. In einer Gesellschaft, in der alle Akteure in einem Begründungsund Legitimationszwang stehen, führt dies fast zwangsläufig zu einer Krise einstmals etablierter Institutionen wie z. B. der Wissenschaften und der Medien (McIntyre 2018; Lewandowsky et al. 2017). Zudem hängen damit Entwicklungen wie der Wertewandel (Lewandowsky et al. 2017) und ein Ende eines Wahrheitskonsenses (Mair 2017) zusammen.

Im Mittelpunkt der meisten Analysen stehen Veränderungen in der Medienlandschaft (Lewandowsky et al. 2017). Dazu zählen das Aufkommen der Aufmerksamkeitsökonomie (Harsin 2018, S. 14ff.), die aufmerksamkeitsstarke Desinformationen und Bullshit begünstigen. Hinzu kommen Veränderungen durch Social Network Sites: von der Fragmentierung der Publika bis hin zu den vielfach behaupteten Filterblasen (Kalpokas 2019). Und schließlich wird die ökonomische Krise des Journalismus (Harsin 2018; McIntyre 2018) angeführt, der immer weniger in der Lage sei, die strategischen Kommunikatoren zu überprüfen. In der Politik werden weitere Ursachen verortet. $\mathrm{Ne}-$ ben einer grundsätzlichen Professionalisierung der strategischen politischen Kommunikation (Harsin 2018) ist dies eine neue Kultur der Unehrlichkeit bei Politikern. Etwas grundsätzlicher wird für viele Länder zudem eine Polarisierung der politischen Landschaft beschrieben, die auf eine wachsende ökonomische Ungleichheit zurückgeht (Lewandowsky et al. 2017). 
Manche Autoren weisen eine Mitschuld an der Wahrheitskrise auch den Erkenntnistheoretikern zu (Hoffjann 2021), die mit nichtrealistischen Positionen dem Relativismus Tür und Tor geöffnet und damit den Vertretern alternativer Fakten eine wissenschaftliche Legitimation geboten hätten: Postfaktische Phänomene seien ein Produkt der postmodernen Theorie (McIntyre 2018), so könnten zum Beispiel die Simulationen und die Hyperrealität von Baudrillard (1978a, 1978b) als die theoretischen Grundlagen einer postfaktischen Politik verstanden werden (d'Ancona 2017): Die Öffentlichkeit habe „adopted bastardized versions of relativism and scepticism derived from academic versions of postmodernism“ (Mair 2017, S. 4). Die gesellschaftlichen Herausforderungen durch aktuelle Desinformationen und postfaktische Politik haben auch im traditionell breiten erkenntnistheoretischen Diskurs der deutschsprachigen Kommunikationswissenschaft Spuren hinterlassen. So wähnen sich konstruktivistische Kommunikationswissenschaftler angesichts von Desinformationen in der Defensive und scheinen sich mitunter an realistische Positionen anzunähern (z. B. Pörksen 2018). In Zeiten offensichtlicher Lügen gibt es mithin eine neue Sehnsucht nach Sicherheiten: „Ich war lange Zeit (Radikaler) Konstruktivist. Heute sehne ich mich nach Wahrheit und Erkenntnis, in einem strikt empirischen Sinne. Ich sehe im Moment nicht, dass ich die irgendwo herbekomme. Wir bräuchten, wenn wir an Wahrheit und Erkenntnis festhalten wollen, an der einen Wirklichkeit, eine Art ,empirischen Journalismus', wie immer der auszusehen hat: datengetrieben, statistisch fundiert. Aber auch Statistiken können manipulieren. Wir kommen aus dem Problem offenbar nicht heraus“ (Weber 2019).

\subsection{Postfaktische Politik, politische Postfakten: Bullshit vs. Lügen}

Wenn postfaktische Politik als neue Ära verstanden wird, ist zu konkretisieren, worin die zentralen Unterschiede zu nicht postfaktischer Politik bestehen. Anders formuliert: Wodurch unterscheidet sich postfaktische Politik von einer faktischen Politik? Dies kann systemtheoretisch begründet erläutert werden. Die Annahme postfaktischer Politik unterstellt im Wesentlichen, dass Wahrheit insbesondere bei öffentlichen politischen Aussagen unwichtiger wird. Wenn die Funktion des politischen Systems die Herstellung kollektiv bindender Entscheidungen ist (Luhmann 1991, S. 159), kann politische Kommunikation in einem weiten Sinne als alle Kommunikationen des politischen Systems und damit Kommunikation im Medium der Macht definiert werden. Da das auch den privaten politischen Diskurs umfasst, muss dies weiter eingegrenzt werden. Im Folgenden soll politische Kommunikation in einem engen Sinne verstanden werden und nur strategische Organisationskommunikation politischer Organisationen umfassen. Strategische Kommunikation kann dabei definiert werden als „purposeful use of communication by an organization to fulfill its mission“ (Hallahan et al. 2007, S. 3).

Die Erwartungen an Wahrheit und Angemessenheit strategischer politischer Kommunikation sind seit jeher widersprüchlich. Daher muss differenziert werden zwischen normativen und kognitiven Erwartungen. Man erwartet normativ die Pünktlichkeit von Zügen, zeigt sich aber lernbereit, indem man bei wichtigen Terminen eine frühere Zugverbindung nimmt. Für die Erwartungen an Politik heißt dies: Man erwartet normativ, dass Politikern die Wahrheit wichtig ist und sie wahrhaftig reden. Werden sie bei einer Lüge ertappt, wird dies skandalisiert, um die Wahrheitsnorm zu restabilisieren. Dieser normativen Erwartungshaltung steht die kognitive Erwartungshaltung gegenüber, die bereits Hannah Arendt betonte: „Wahrhaftigkeit zählte niemals zu den politischen Tugenden“ (Arendt 2013, S. 8).

Welche „Art“ von Wahrheit wird von der Politik erwartet? In einer systemtheoretischen Betrachtung „produziert“ Wissenschaft mit ihrem Code wahr vs. unwahr Wahr- 
heit, wahr kann also als rein systeminterne Zuschreibungen verstanden werden (Luhmann 1994; Kaldewey 2013). Während in der organisierten Wissenschaft u. a. nach konsentierten Prüfverfahren über wahr und unwahr entschieden wird, kann in wissenschaftlicher Alltagskommunikation jede mit der Unterscheidung operieren und damit wissenschaftlich kommunizieren (Luhmann 1994). Alltägliche wissenschaftliche Kommunikation unterscheidet sich von organisierter in der Regel dadurch, dass sie in der Wissenschaft, aber häufig auch in anderen Funktionssystemen, weniger anschlussfähig ist (Haacke 2019).

Welche Relevanz haben Wahrheit und damit die Wissenschaft für die Politik? Politik nimmt Bezug auf Wissenschaft bzw. Wahrheit, um politische Entscheidungen zu legitimieren (Haacke 2019). Diese Bezugnahme erfolgt in der Regel instrumentell: Politik setzt wissenschaftliche Beiräte ein oder bezieht sich auf (passende) wissenschaftliche Studien, wenn es ihren Zielen nützt. Eine faktische Politik ist also durch (implizite) Wahrheitsbehauptungen geprägt, wonach Aussagen einer wissenschaftlichen Überprüfung standhalten würden - also auch nach Anwendung anerkannter Testverfahren als wahr bezeichnet würden. Ein solches systemtheoretisches Verständnis von Wahrheit ist kompatibel mit allen relevanten erkenntnistheoretischen Ansätzen, von realistischen über konstruktivistische bis hin zu non-dualistischen Positionen (Hoffjann 2013; Kaldewey 2013).

Die Pluralität wissenschaftlicher Erkenntnisse hat auch jenseits des organisierten Wissenschaftsbetriebs das Bewusstsein gestärkt, dass wissenschaftliche Befunde kontingent sind bzw. auch organisierte Wissenschaft sich irren kann (Beck 1986). Die Folge: In der organisierten Wissenschaft produzierte Wahrheit wird in der Politik immer häufiger zur Meinung transformiert. Während die strukturellen Kopplungen zwischen Politik und Wissenschaft in einer faktischen Politik enger sind, sind sie in einer postfaktischen Politik loser. In einer postfaktischen Politik wird der Wahrheitsbezug mithin unwichtiger für politischen Erfolg (Haacke 2019).

Postfaktische Aussagen sind damit keine Lügen, sondern Bullshit. Während sich eine postfaktische Politikerin nicht für Wahrheit interessiert, sagt eine Lügnerin etwas wider besseres Wissen - nimmt also negativ Bezug auf die Wahrheit (Frankfurt 2006; van Dyk 2017; Haacke 2019). „Ideal-typical bullshit is immune to any attempt to scrutinize a claim against the empirical record. Confronting the bullshitter with 'the facts' is unlikely to be a successful argumentative strategy. This, in a nutshell, is what makes bullshit so much more dangerous and socially corrosive than lying." (Hopkin \& Rodamond 2018, S. 643) Deshalb erscheint es plausibel, Politiker wie Donald Trump oder Boris Johnson eher als Bullshitter denn als notorische Lügner zu bezeichnen. Gleichwohl ist zu erwarten, dass auch Lügen einer postfaktischen Politik häufiger unterstellt werden.

Während Postfakten bzw. Bullshit Wahrheit ignorieren, eine Lügnerin von der Unwahrheit ihrer Aussage ausgeht, liegen explizite Bewertungen hierzu quer. Eine Kommunikatorin kann mit einer völlig übertriebenen Bewertung wie eine Lügnerin eine Täuschungsabsicht verfolgen, oder aber sie interessiert sich dabei wie eine Bullshitterin nicht für die Wahrheit. Solche Bewertungen sind für Rezipientinnen einerseits eine erhebliche Handlungsentlastung, andererseits aber auch besonders riskant (Kohring 2004). Es ist zu vermuten, dass strategische politische Kommunikation hier eine größere „Narrenfreiheit“ genießt: Weil Rezipientinnen um den Selbstdarstellungscharakter wissen, dürften z. B. kleinere Übertreibungen geduldet werden und Kommunikatorinnen wiederum diese Duldung unterstellen. So zeigt auch Kohring in seiner Studie zum Vertrauen in Journalismus (2004, S. 252), dass die Risikowahrnehmung in der Bewertungsdimension geringer ist als in den drei anderen Dimensionen. Trotz des kategorialen Unter- 
schiedes zwischen dem fremddarstellenden Journalismus und der selbstdarstellenden strategischen politischen Kommunikation ist zu erwarten, dass beide Formen sich hierin nicht unterscheiden. Daraus folgt die erste Forschungsfrage:

RQ 1: Wie bewerten Politikerinnen, Pressesprecherinnen und Journalistinnen die Verbreitung und Akzeptanz von Lügen und Bullshit in der strategischen politischen Kommunikation?

Zur (unterstellten) Verbreitung von Postfakten und Lügen in der Politik liegen bislang kaum empirische Befunde vor. Dies überrascht angesichts der Prominenz der Diskurse zur postfaktischen Politik, zum Vertrauen in Politik bzw. Politikerinnen (Überblicke in Citrin \& Stoker 2018; Zmerli \& van der Meer 2017) sowie zu Wirkungen von Desinformationen in Medien (z. B. Arendt et al. 2019; Wagner \& Boczkowski 2019) bzw. zu Einstellungen wie der Medienglaubwürdigkeit. Zur wahrgenommenen Ehrlichkeit bzw. unterstellten Verbreitung von Desinformationen liegen lediglich singuläre Befunde vor. In einer norwegischen Wählerinnenbefragung nehmen mehr Befragte ihre Politikerinnen als unehrlich wahr (Enli \& Rosenberg 2018, S. 4). Für Deutschland weist der „Reuters Institute Digital News Report“ (Hölig \& Hasebrink 2020, S. 36) aus, dass 25 Prozent der Bürgerinnen Falschmeldungen am ehesten von deutschen politischen Organisationen befürchten, etwas mehr als von Aktivistengruppen sowie von ausländischen Regierungen. Die sowohl in Wissenschaft als auch in der Öffentlichkeit breit geführte Diskussion (Schwaiger \& Eisenegger 2021) zur postfaktischen Gesellschaft sowie zum Bullshit von Politikern wie Trump und Johnson lässt erwarten, dass Bullshit verbreiteter ist bzw. als verbreiteter wahrgenommen wird als Lügen. Da Postfakten die Wahrheitsnorm ignorieren, aber im Gegensatz zur Lüge kein bewusster Verstoß gegen sie sind (Frankfurt 2006; Haacke 2019), ist zudem davon auszugehen, dass sie akzeptierter sind. Entsprechend lauten die ersten beiden Hypothesen:

H 1.1: Bullshit ist weiter verbreitet als die Lüge.

H 1.2: Bullshit ist akzeptierter als die Lüge.

\section{2 „Bürger" und „Akteure“ einer postfaktischen Politik}

Es fällt auf, dass die postfaktische Politik zumeist in theorieorientierten Beiträgen erläutert und ausgeführt wird. Überraschen kann dies aus dem Grund nicht, da Kategorien wie Wahrheit, Lüge und Bullshit empirische Sozialforschung in der Regel vor große Probleme stellen. Daher sind (vermeintliche) Belege für eine postfaktische Politik zumeist anekdotischer Natur. Empirische Befunde liegen hierzu, außer den bereits genannten, bislang kaum vor.

Für die Existenz postfaktischer Politik ist aber weniger das Vorhandensein bzw. die Verbreitung des Bullshitting wichtig, sondern vielmehr die Unterstellung desselben (Higgins 2016). Der reflexive Charakter postfaktischer Politik impliziert, dass man postfaktische Politik nicht nur selbst unterstellt, sondern diese Unterstellung auch anderen unterstellt. Wer grundsätzlich Politikern die Irrelevanz der Wahrheit unterstellt und diese Unterstellung auch anderen weithin unterstellt, wähnt sich in einer postfaktischen Politik. Dies entspricht der Kongruenzdimension im Koorientierungsmodell, die die Beziehung zwischen den eigenen Vorstellungen sowie den der anderen Seite unterstellten Vorstellungen beschreibt (McLeod \& Chaffee 1972). Wer allerorten postfaktische Aussagen unterstellt, sieht sich damit als „Bürger“ einer postfaktischen Politik. Zudem beeinflussen solche Unterstellungen und Unterstellungsunterstellungen auch das Verhal- 
ten z. B. von Journalisten, Pressesprechern und Politikern, weil diese ihr Kollegen- bzw. Adressatenbild konkretisieren.

Man kann davon überzeugt sein, in einer postfaktischen Demokratie zu leben, dennoch postfaktische Politiker ablehnen. Dazu soll zwischen der erlebenden und handelnden Perspektive unterschieden werden: Während Luhmann beim Erleben Selektionsleistungen nicht dem fokalen System, sondern dessen Umwelt zuschreibt (Luhmann 1993, S. 68f.), rechnet Handeln die Selektivität dem fokalen System selbst zu. Da sich bei sensiblen Themen wie Bullshit und Lüge in hohem Maße das Problem sozialer Erwünschtheit (King \& Bruner 2000) stellt, ist es methodisch problematisch zu fragen, ob Politiker und Pressesprecher Postfakten bzw. Lügen nutzen. Wird ein Verhalten hingegen als legitim bewertet, kann daraus zwar nicht ein entsprechendes Verhalten sicher geschlossen werden, ist aber wahrscheinlicher (Lamnek et al. 2013, S. 153). Zudem unterstützt eine solche Person eine postfaktische Politik, indem sie sie in Gesprächen nicht verurteilt bzw. sogar noch legitimiert und ein solches Verhalten damit fördert; Personen, die postfaktische Politik als legitim bewerten, können daher als „postfaktische Akteure“ bezeichnet werden. Daraus folgt die zweite Forschungsfrage:

\section{RQ 2: Wie hoch ist der Anteil an Politikern, Journalisten und Pressesprechern, die sich als Teil einer postfaktischen Politik verstehen?}

Die wenigen vergleichenden Befragungen von Journalisten und Politikern bzw. Pressesprechern (z. B. Hoffjann \& Lohse 2016; Pfetsch \& Mayerhöffer 2011) haben Aspekte der Glaubwürdigkeit oder Wahrhaftigkeit bislang nicht untersucht, zeigen aber recht eindeutig, dass Fehlverhalten mehrheitlich auf der jeweils anderen Seite erkannt wird. Mit Blick auf eine postfaktische Politik, die Fehlverhalten auf der Seite der Politiker verortet, folgt damit die dritte Hypothese:

\section{H 2.1: Journalisten sehen sich eher als „postfaktische Bürger" als Politiker und Presse- sprecher.}

In der Literatur werden Politiker wie Trump, Johnson und Bolsonaro vielfach sowohl als populistische Politiker (z. B. Gugushvili et al. 2020; Şahin 2021) als auch als postfaktische Politiker bezeichnet (z. B. MacKenzie \& Bhatt 2020). Es gibt jedoch erstaunlich wenige Verbindungen zwischen den Diskursen zur populistischen und zur postfaktischen Politik. Trotz zahlreicher vorliegender Definitionen zum Populismus (Überblick in Pappas 2016) wird verschiedentlich das Fehlen einer elaborierten und systematisierenden Theorie und Definition zum Populismus beklagt (Müller 2016; Gil de Zúñiga et al. 2020). Als zentrale Merkmale des Populismus können mit Müller (2016) der AntiElitismus, antipluralistische Annahmen und Formen der Identitätspolitik verstanden werden. Engesser et al. (2017) leiten aus den Definitionen vier Dimensionen populistischer Kommunikation ab: Inhalte, Stil, Ziele sowie Akteure populistischer Kommunikation. Als mögliche Stilformen benennen sie u. a. Negativität, Emotionalisierung, Vereinfachung, Dramatisierung, Polarisierung, Moralisierung und eine vulgäre Sprache.

Bei den Stilformen wäre am ehesten eine postfaktische Politik zu verorten, hier zeigt sich aber einmal mehr das Problem postfaktischer Politik für die empirische Forschung. Während die anderen genannten Stilformen in Inhaltsanalysen vielfach erforscht wurden (z. B. Maurer \& Jost 2020; Spieß et al. 2020), ist dies für Kategorien wie Wahrheit, Lüge oder Bullshit nicht möglich. Dies mag ein Grund dafür sein, dass sich in der Forschung nur wenige elaborierte Ausführungen dazu finden lassen, inwieweit postfaktisches Verhalten ein originäres Merkmal des Populismus ist. Während vereinzelt davon ausgegangen wird, dass eine postfaktische Politik kein originäres Merkmal des Populismus ist (z. B. Hopkin \& Rosamond 2018), erkennen mehrere Autoren hier durchaus einen Zu- 
sammenhang, der insbesondere mit dem Anti-Elitismus sowie den antipluralistischen Annahmen begründet wird (z. B. Gil de Zúñiga et al. 2020; Fraune \& Knodt 2018; Gugushvili et al. 2020; Hameleers \& Minihold 2020; Tumber \& Waisbord 2021). Für Deutschland sind für die AfD in vielen Untersuchungen populistische Merkmale nachgewiesen worden (z. B. Lewandowsky et al. 2016; Maurer \& Jost 2020; Spieß et al. 2020). Daraus ergeben sich die vierte und fünfte Hypothese:

H 2.2: Abgeordnete und Pressesprecher der AfD verstehen sich eher als „postfaktische Bürger" als andere Parteienvertreter.

H 2.3: Abgeordnete und Pressesprecher der AfD verstehen sich eher als „postfaktische Akteure" als andere Parteienvertreter.

\subsection{Journalistische Reaktionen auf postfaktische Politikerinnen}

Wie bewerten Journalistinnen Politikerinnen, denen sie Bullshit unterstellen? Sie stehen vor einem vergleichbaren Problem wie die Wissenschaft: Jenseits erkenntnistheoretischer Überlegungen ließen sich ihrer Meinung nach unwahre Aussagen durch einen Vergleich z. B. mit offiziellen Statistiken im Sinne eines Basiskonsenses finden (Mitterer 1992, 2001). Ob der Politikerin die Wahrheit egal war, sie bewusst gelogen hat oder sich lediglich irrte, bleibt außer bei Geständnissen eine Zuschreibungsleistung. So zählte auch die Washington Post (2020) nicht die Lügen von Donald Trump, sondern bis zur Wahl 2020 knapp 30.000 falsche oder irreführende Aussagen während seiner Amtszeit als Präsident. Für die Forschung kommt als zweites Problem hinzu, dass auch journalistische Recherchen und Faktenprüfung selbst eine Black Box sind (Godler 2020, S. 170).

Ältere Studien, die vor der intensiven Auseinandersetzung mit Phänomenen wie Fake News und postfaktischer Politik entstanden sind, zeigen, dass Journalistinnen der PR insgesamt kritisch gegenüberstehen (z. B. Weischenberg et al. 2006; Bentele \& Seidenglanz 2004). Der vermutete Zusammenhang von Rollenselbstverständnis und Einstellungen zur PR hat sich in diesen Studien jedoch nicht bestätigt. Eine kritische bzw. investigative Journalistin steht PR grundsätzlich nicht skeptischer gegenüber als vermittelnde oder unterhaltungsorientierte Kolleginnen (Scholl 2018; ähnlich Seidenglanz 2015). In einigen wenigen aktuelleren Studien ist untersucht worden, wie Journalismus auf die Herausforderung einer postfaktischen Politik reagiert (z. B. Godler 2020). Dabei ist unter anderem gefragt worden, wie er mit Ungewissheit umgeht (Eldridge \& Bødker 2018) und wie er seine eigene Verantwortung beim Umgang mit postfaktischen Politikern sieht. Die Ergebnisse: Die eigene Verantwortung wird nur selten thematisiert (Tandoc et al. 2019), überwiegend wird sie außerhalb des Journalismus verortet (McDevitt \& Ferrucci 2018). Die konkreten Reaktionen der Journalistinnen im Umgang mit postfaktischen Politikerinnen sind in diesen Studien allerdings nicht untersucht worden. Daraus folgt die dritte Forschungsfrage:

\section{$R Q$ 3: Wie reagieren Journalistinnen auf Politikerinnen, denen sie eine feblende Rele- vanz der Wabrbeit unterstellen?}

In den vergangenen Jahren ist - jenseits empirischer Studien - vielfach herausgestellt worden (z. B. Waisbord 2018; Carlson 2018; Romano 2017), dass eine postfaktische Politik eine Bedrohung für den Journalismus darstelle und er als Reaktion hierauf umso mehr an der Wahrheitsnorm festhalte, je verbreiteter er Phänomene wie Desinformation und Postfakten wahrnehme. In dieser Perspektive ist eine kritische Berichterstattung über postfaktisches Gebaren grundsätzlicher als Abwehrstrategie gegenüber einer übersteuernden Politik, die zumal als illegitim bewertete Methoden nutzt, und damit gleich- 
zeitig als Legitimationsstrategie gegenüber den journalistischen Publika zu interpretieren. Vor dem Hintergrund dieser Überlegungen ist zu erwarten, dass Journalistinnen, die vielen Politikerinnen eine fehlende Relevanz der Wahrheit unterstellen, dies eher kritisieren als Journalistinnen, die noch an eine weitgehende Einhaltung der Wahrheitsnorm glauben. Umgekehrt ist zu erwarten, dass Journalistinnen, die Methoden wie Bullshit und Lügen legitimieren, dies eher als „Teil des Spiels“ ansehen als Journalistinnen, die solche Praktiken verurteilen. Die letzten beiden Hypothesen lauten entsprechend:

H 3.1: Journalistinnen, die sich eher als „postfaktische Bürgerinnen"verstehen, kritisieren mögliche Täuschungen und Lügen eher als Journalistinnen, die sich eher "faktische Bürgerinnen" seben.

H 3.2: Journalistinnen, die sich eher als "faktische Akteurinnen"verstehen, kritisieren mögliche Täuschungen und Lügen eher als Journalistinnen, die sich eher als "postfaktische Akteurinnen" seben.

\section{Methode}

Zur Beantwortung der Forschungsfragen wurde eine Onlinebefragung unter den $\mathrm{Ab}$ geordneten des Deutschen Bundestages $(\mathrm{MdB})$ und aller deutschen Landtage $(\mathrm{MdL})$, den Mitgliedern der Bundes- und aller Landespressekonferenzen sowie von Pressesprechern bzw. Öffentlichkeitsarbeitern in Parteien, Fraktionen und Ministerien auf Bundes- und Länderebene durchgeführt. Die Befragten befassen sich somit alle beruflich mit der gleichen Thematik (Bundespolitik bzw. Landespolitik im jeweiligen Bundesland) und haben persönlich miteinander zu tun. Die Studie knüpft damit in Teilen an eine Befragung von 2015 an (Hoffjann \& Lohse 2016). Zusätzlich zum damaligen Sample wurden dieses Mal Pressesprecher befragt, da ihnen bei der strategischen politischen Kommunikation eine zentrale Rolle zukommt. Da die Studie damals deutlich gezeigt hat, dass es bei Fragen der Beziehungen von Abgeordneten und Journalisten keine signifikanten Unterschiede zwischen bundes- und landespolitischen Akteuren gibt, wird bei den nachfolgenden Auswertungen nicht mehr zwischen Landes- und Bundesebene unterschieden.

Die Befragten ordneten sich zu Beginn des Onlinefragebogens einer der drei Gruppen zu. Neben vollständig identischen Fragen gab es weitgehend gleiche Fragen, bei denen nur die Formulierungen an Politiker, Journalisten oder Pressesprecher angepasst wurden, sowie spezifische Fragen, bei denen alle Items angepasst wurden (z. B. zum Rollenselbstverständnis) oder die nur der jeweiligen Gruppe gestellt wurden. Die nachfolgend vorgestellten Befunde sind nur ein kleiner Ausschnitt aus der Gesamtstudie. Der Fragebogen gliederte sich in die Abschnitte Rollenselbstverständnis, Orientierung an spezifischen Gruppen, Beziehungskultur, allgemeine Aussagen zur politischen Kommunikation, Verbreitung und Akzeptanz von Praktiken in der politischen Inszenierung bzw. zu Aussagen von Politikern sowie des Politikjournalismus, zur unterstellten Verbreitung von Annahmen zum Wahrheitsgehalt von Aussagen von Politikern sowie abschließend Fragen zur Berufserfahrung, beruflichen Rolle und zur Partei (bei Politikern und Pressesprechern). Alle Fragen und Items, die zur Beantwortung der Forschungsfragen relevant sind, wurden selbst entwickelt, da zu diesen Themen noch keine vergleichbaren Studien vorlagen. Die Fragebögen wurden nach einem Pretest überarbeitet. Die Operationalisierung der Hypothesen sowie die Herleitung für die Typenbildung ${ }^{2}$ werden im nächsten Kapitel detailliert erläutert.

2 Aufgrund der komplexen Bedingungen für die Typenbildung von postfaktischen bzw. faktischen „Bürgern“ bzw. „Akteuren“ variieren nachfolgend teilweise die n-Werte. 
Während die Kontaktdaten der Abgeordneten öffentlich zugänglich sind und damit eine Vollerhebung möglich war, stellten sieben Landespressekonferenzen keine Mitgliederdaten zur Verfügung, sondern leiteten eine Einladungsmail mit einem offenen Umfragelink an ihre Mitglieder weiter. Bei den restlichen neun Landespressekonferenzen wurden die Kontaktdaten aller Mitglieder (zum Teil kostenpflichtig) zur Verfügung gestellt. Die Namen und Kontaktdaten der Pressesprecher bzw. Öffentlichkeitsarbeiter wurden per Internetrecherche erhoben. Die Erhebung hatte den in Tabelle 1 gezeigten Rücklauf.

Tabelle 1: Übersicht Gesamtsample und Rücklauf

\begin{tabular}{lccc}
\hline & Gesamtsample & Rücklauf & Rücklaufquote \\
\hline Abgeordnete & 2.550 & 342 & $13,41 \%$ \\
Journalisten & 1.420 & 231 & $16,27 \%$ \\
Pressesprecher & 687 & 185 & $26,93 \%$ \\
Gesamt & 4.657 & 758 & $16,28 \%$ \\
\hline
\end{tabular}

Die Daten wurden im Zeitraum vom 14.10.2020 bis zum 04.01.2021 erhoben. Die Rücklaufquote für die Journalisten und Abgeordneten liegen einerseits etwas unter anderen Befragungen (z. B. Hoffjann \& Lohse 2016; Nuernbergk \& Schmidt 2020; Ohliger \& Vögele 2020; Pfetsch \& Mayerhöffer 2011; Pontzen 2013). Möglicherweise hat die Vielzahl der Befragungen dazu geführt, dass die Bereitschaft von Abgeordneten zur Teilnahme an Studien seit Jahren kontinuierlich sinkt (Best et al. 2010). Andererseits ist eine Rücklaufquote von etwa 16 Prozent im Hinblick auf die Rekrutierung via Mailinglisten aus Datenbanken als ausreichend zu bewerten (Batinic \& Moser 2005).

Die Stichprobe entspricht bei der Fraktions- und Parteizugehörigkeit bei den meisten Parteien der Zusammensetzung der Grundgesamtheit: SPD (Anteil Grundgesamtheit: 23,7 \% / Anteil Stichprobe: 20,5 \%), AfD (12,1\% / 11,9\%), FDP (7,9\% / 9,6 \%) und Die Grünen (13,4 \% / 12,6 \%). Während die CDU/CSU unterrepräsentiert ist (31,6 \% / $21,2 \%)$, sind Vertreter der Linken überrepräsentiert (8,1 \% / 15,7 \%). Für die befragten Pressesprecher und Journalisten liegen keine ähnlichen Daten (z. B. Altersstruktur von BPK-Mitgliedern) vor. Das Durchschnittsalter aller Befragten beträgt 50,6 Jahre, 70,7 \% der Befragten waren männlich. Somit nähern sich die Umfragedaten - soweit Informationen zu den Grundgesamtheiten vorliegen - an die realen Verhältnisse an, können streng genommen jedoch nicht als repräsentativ gelten.

\section{Ergebnisse}

\subsection{Verbreitung und Akzeptanz von Bullshit und Lüge}

Wie verbreitet und akzeptiert sind Bullshit und Lüge in der strategischen politischen Kommunikation? Journalistinnen, Politikerinnen und Pressesprecherinnen sind einerseits nach der Verbreitung, andererseits nach der Legitimität verschiedener Praktiken in der Politik gefragt worden. Zusätzlich ist nach der Verbreitung und Legitimität von Übertreibungen gefragt worden, die quer liegen zu Aspekten wie Wahrhaftigkeit, Lüge oder Bullshit. Übertreibungen können sowohl mit Täuschungsabsichten, einem Desinteresse für Wahrheit als auch - im Falle leichter Übertreibungen - mit einem Wahrhaftigkeitsanspruch vereinbar sein. Daher überrascht es nicht, dass Übertreibungen von 
Tabelle 2: Verbreitung und Legitimität von Übertreibungen, Bullshit und Lüge in der Politik (Angaben in \%)

\begin{tabular}{ccc}
$\begin{array}{c}\text { Journalistinnen } \\
(\mathrm{n}=185-194)\end{array}$ & $\begin{array}{c}\text { Politikerinnen } \\
(\mathrm{n}=290-294)\end{array}$ & $\begin{array}{c}\text { Pressesprecherinnen } \\
(\mathrm{n}=152-157)\end{array}$ \\
\hline
\end{tabular}

„Wie verbreitet sind Ihrer Einschätzung nach die folgenden Praktiken in der Politik?"

(„sehr verbreitet“ / „verbreitet“; 5er Skala)

Übertreibungen

(„Übertreibungen (z. B. bei

84,5

76,9

70,7

Bewertungen)“)

Bullshit

(„Ergänzen von ungeprüften Aussagen, die wahr sein könnten, um die These

35,9

einer Aussage zu stützen.“)

Lügen

(„Erfinden von Aussagen, um die These

14,3

21,8

5,1

eines Statements zu stützen.“)

„Wie legitim sind Ibrer Einschätzung nach die folgenden Praktiken in der Politik?“ („völlig legitim“ / „eher legitim“; 5er Skala)

$\begin{array}{lccc}\text { Übertreibungen } & 30,9 & 30,9 & 42,8 \\ \text { Bullshit } & 6,5 & 4,1 & 3,9 \\ \text { Lügen } & 2,7 & 0,7 & 0,7\end{array}$

allen Gruppen als die Praktik mit der größten Verbreitung und Akzeptanz eingeschätzt wurden.

Journalistinnen nehmen Bullshit als verbreiteter wahr, als dies Politikerinnen und Pressesprecherinnen tun, während Politikerinnen Lügen als verbreiteter ansehen als die beiden anderen Gruppen. Die Einschätzungen der Pressesprecherinnen sind dabei auffällig, da sie die Verbreitung von Lügen als deutlich geringer einschätzen. Relativ einig sind sich alle Gruppen in dem Urteil, dass Lügen illegitim seien, wenngleich Journalistinnen dies als noch etwas legitimer erachten als die beiden anderen Gruppen (Tabelle 2).

Über alle Gruppen hinweg zeigt sich, dass Bullshit $(\mathrm{MW}=2,94 ; \mathrm{SD}=1,119)$ verbreiteter ist als die Lüge ( $M W=2,19 ; \mathrm{SD}=1,143)$ (Pearsons $\mathrm{r}=0,658 ; \mathrm{p}<0,01)$. Darüber hinaus wird Bullshit $(\mathrm{MW}=1,7 ; \mathrm{SD}=0,840)$ als legitimer eingeschätzt als Lügen $(\mathrm{MW}=1,12 ; \mathrm{SD}=0,485)$ (Pearsons $\mathrm{r}=0,422, \mathrm{p}<0,01)$. Es bestätigt sich damit die Erwartung, dass ein bewusster Verstoß gegen die Wahrheit weniger akzeptiert ist als ein gleichgültiges Verhältnis zur Wahrheit.

\section{2 „Bürger" und „Akteure“ einer postfaktischen Politik}

In der Studie wird differenziert zwischen der erlebenden Perspektive, in der Politikern eine weitgehende Gleichgültigkeit gegenüber der Wahrheit unterstellt wird (nachfolgend: „postfaktische Bürger“), und einer handelnden Perspektive, in der dies für legitim erachtet wird (nachfolgend: „postfaktische Akteure“). Wie hoch ist der Anteil an Politikern, Pressesprechern und Journalisten, die sich selbst als „Bürger“ einer postfaktischen Politik wahrnehmen? Dies ist mit mehreren Variablen operationalisiert worden. Dem reflexiven Charakter postfaktischer Politik wurde dadurch Rechnung getragen, dass zur Aussage „Man liest ja heute mitunter Behauptungen wie die Folgende: ,Politi- 
kerinnen und Politikern ist der Wahrheitsgehalt ihrer Aussagen weitgehend egal."“ nicht nur nach der eigenen Bewertung der Befragten gefragt wurde, sondern auch danach, welche Einschätzung sie (anderen) Journalisten, Politikern und Bürgern unterstellen. ${ }^{3}$ In Anlehnung an die oben genannten Definitionsmerkmale wurde zudem gefragt, als wie weit verbreitet die Emotionalisierung in der Politik wahrgenommen wird. ${ }^{4}$ Als eher „postfaktische Bürger“ gelten Befragte mit einem Mittelwert von mehr als 3,0.5

Die deskriptive Betrachtung deutet an, dass die Berufsgruppen (Journalist, Politiker, Pressesprecher) innerhalb der Kategorie „Bürger“ einer eher postfaktischen und einer eher faktischen Politik in etwa gleich groß sind (Tabelle 3). Bei den einzelnen Gruppen zeigt sich, dass Politiker in einem höheren Maße als Pressesprecher und Journalisten angeben, dass eine emotionalisierende Politik verbreitet ist. Rund 55 Prozent der Politiker glauben demnach an eine postfaktische Politik. Pointiert formuliert lässt die deskriptive Betrachtung den Schluss zu, dass sowohl Journalisten als auch Pressesprecher häufiger an das Gute in Politikern glauben als diese selbst.

Die einfaktorielle Varianzanalyse (ANOVA) zeigt, dass sich der Anteil der Journalisten unter den postfaktischen Bürgern nicht statistisch signifikant von den Politikern und Pressesprechern unterscheidet $(\mathrm{F}(2,662)=2,412 ; \mathrm{p}=0,09)$. Folglich kann Hypothese 2.1 nicht angenommen werden.

Das Konstrukt des „postfaktischen Akteurs“ wurde mit drei Variablen operationalisiert: die Legitimitätseinschätzungen zur Emotionalisierung als politische Praxis, zum Bullshitting und zur Lüge. ${ }^{6}$ Als eher „postfaktische Akteure“ gelten Befragte mit einem Mittelwert von mehr als 3,0.7 Bei den „Akteuren“ gibt es kaum Unterschiede zwischen den drei Gruppen: Mehr als neun von zehn Befragte können als eher „faktische Akteure" bezeichnet werden.

Welche Unterschiede lassen sich zwischen Politikern und Pressesprechern unterschiedlicher Parteien finden? Dazu wurden Abgeordnete und Pressesprecher zu „Vertretern einer Partei“ zusammengefasst. Die Parteizuordnung bei Pressesprechern erfolgte über die Frage, für welche Partei oder Fraktion sie arbeiten bzw. welcher Partei ihr Minister angehört. Auch wenn Sprecher eines Ministeriums keine Parteiinteressen

3 „Wie bewerten Sie selbst diese Aussage?“; „Was glauben Sie, wie bewerten (andere) Journalistinnen und Journalisten dies?"; „Was glauben Sie, wie bewerten (andere) Politikerinnen und Politiker dies?"; „Was glauben Sie, wie sehen Bürgerinnen und Bürger dies?"; Verwendung einer fünfstufigen Likert-Skala von $1=$,stimme(n) überhaupt nicht zu“ bis $5=$ „stimme(n) voll und ganz zu“.

4 „Öffentliche Auftritte in der Politik werden heute vielfach aufwändig inszeniert. Das kann auf unterschiedliche Art und Weise geschehen. Wie weit verbreitet sind Ihrer Meinung nach die folgenden Praktiken?“; u. a. wurde gefragt nach der „Nutzung emotionaler Elemente (Emotionalisierung)“; Verwendung einer fünfstufigen Likert-Skala von $1=$ „kaum verbreitet“ bis $5=$ „sehr verbreitet“.

5 Verwendung einer fünfstufigen Likert-Skala von $1=$ „kaum verbreitet“ bis $5=$ „sehr verbreitet“ bzw. 1 = „stimme überhaupt nicht zu“ bis $5=$ „stimme voll und ganz zu“.

6 „Wie legitim sind Ihrer Meinung nach die folgenden Praktiken in der Politik?“; im Kontext von Inszenierungspraktiken wurde u. a. gefragt nach der Legitimität der „Nutzung emotionaler Elemente (Emotionalisierung)“; im Kontext von aussagenbezogenen Praktiken wurde u. a. gefragt nach der Legitimität von: „Ergänzen von ungeprüften Aussagen, die wahr sein könnten, um die These einer Aussage zu stützen“ (Bullshit) sowie „Erfinden von Aussagen, um die These eines Statements zu stützen“ (Lüge); Verwendung einer fünfstufigen Likert-Skala von 1 = „nie legitim“ bis 5 = „völlig legitim“.

7 Verwendung einer fünfstufigen Likert-Skala von $1=$ „nie legitim“ bis 5 = „völlig legitim“. 
Tabelle 3: Anteil an „Bürgern“ und „Akteuren“ einer postfaktischen Politik (Angaben in \%)

\begin{tabular}{lccc}
\hline & $\begin{array}{c}\text { Journalisten } \\
(\mathrm{n}=170-189)\end{array}$ & $\begin{array}{c}\text { Politiker } \\
(\mathrm{n}=258-288)\end{array}$ & $\begin{array}{c}\text { Pressesprecher } \\
(\mathrm{n}=141-150)\end{array}$ \\
\hline $\begin{array}{l}\text { Eher „Bürger“ einer postfaktischen } \\
\begin{array}{l}\text { Politik } \\
\text { Eher „Bürger“ einer faktischen Politik }\end{array}\end{array}$ & 44,7 & 55,4 & 49,6 \\
\hline $\begin{array}{l}\text { Eher postfaktische „Akteure“ } \\
\text { Eher faktische „Akteure“ }\end{array}$ & 65,3 & 44,6 & 50,4 \\
\hline
\end{tabular}

vertreten (sollen), so erscheint die Zuordnung wegen des besonderen Vertrauensverhältnisses zwischen Ministern und ihren Sprechern plausibel.

Die deskriptive Auswertung zeigt, dass mit knapp 90 Prozent der Anteil „postfaktischer Bürger“ bei der AfD mit Abstand am höchsten ist, nur vier von 36 AfD-Vertretern sehen sich als „faktische Bürger“ (Tabelle 4). Es wurde eine einfaktorielle ANOVA berechnet um zu untersuchen, ob Abgeordnete und Pressesprecher der AfD eher postfaktische Bürger als andere Parteienvertreter sind. Die Gruppen unterscheiden sich dabei statistisch signifikant $(\mathrm{F}(1,439)=38,456 ; \mathrm{p}<0,001)$. In der Folge kann Hypothese 2.2 angenommen werden. Es zeigt sich also, dass Politiker und Pressesprecher, die sich der AfD zuordnen, sich eher als „postfaktische Bürger“ sehen als Vertreter anderer Parteien. Dies bedeutet, dass AfD-Vertreter häufiger als Vertreter anderer Parteien (im Mittel) angeben, dass Emotionalisierung weit verbreitet ist und sie selbst der Meinung sind, sowie diese Meinung auch (anderen) Politikern, Journalisten und Bürgern unterstellen, dass Politikern der Wahrheitsgehalt ihrer Aussagen weitgehend egal ist.

Deskriptiv zeigt sich (vgl. Tabelle 4) außerdem, dass der geringste Anteil eher „postfaktischer Bürger“ mit 40 Prozent bei den Unionsvertretern zu finden ist und er damit etwas niedriger ist als bei Vertretern von SPD, FDP und Grünen. Auffallend ist zudem der hohe Anteil „postfaktischer Bürger“ bei den Linken mit 67 Prozent. Hier ließen sich möglicherweise Bezüge zu Studien herstellen, die populistische Merkmale im linken Parteienspektrum untersuchen (z. B. Lewandowsky et al. 2016).

Tabelle 4 deutet an, dass bei der AfD und mit etwas Abstand bei den Linken die meisten „postfaktischen Akteure“ vertreten sind, die entsprechende Praktiken legitimieren. Mehr Vertreter innerhalb der AfD als anderer Parteien finden Emotionalisierung, Bullshitting und Lügen - im Mittel - legitim. Insgesamt zeigt sich aber, dass auch 90 Prozent der AfD-Vertreter Emotionalisierung, Bullshitting und Lügen für überwiegend illegitime Methoden halten. Die einfaktorielle Varianzanalyse (ANOVA) konnte diese deskriptiven Tendenzen zwischen den postfaktischen Akteuren der AfD und anderen Parteien nicht statistisch signifikant bestätigen $(F(1,440)=0,548 ; p=0,460)$. In der Folge kann Hypothese 2.2, dass Abgeordnete und Pressesprecher der AfD eher postfaktische Akteure als andere Parteienvertreter sind, nicht angenommen werden.

Die Ergebnisse deuten insgesamt an, dass eine knappe Mehrheit von Abgeordneten und Pressesprechern zwar eine Wahrheitskrise in der Politik wahrnimmt, dass aber eine sehr deutliche Mehrheit dies verurteilt. Mit anderen Worten: Fast alle Befragten, die sich in einer postfaktischen Demokratie wähnen, sind darüber nicht glücklich. 
Tabelle 4: Anteil an „Bürgern“ und „Akteuren“ einer postfaktischen Politik nach Parteien (Politikern und Pressesprechern; Angaben in \%)

\begin{tabular}{lcccccc}
\hline & $\begin{array}{c}\text { CDU/ } \\
\text { CSU } \\
(\mathrm{n}=85)\end{array}$ & $\begin{array}{c}\text { SPD } \\
(\mathrm{n}=74)\end{array}$ & $\begin{array}{c}\text { Bündnis 90/ } \\
\text { Die Grünen } \\
(\mathrm{n}=54)\end{array}$ & $\begin{array}{l}\text { Die Linke } \\
(\mathrm{n}=52)\end{array}$ & $\begin{array}{c}\text { FDP } \\
(\mathrm{n}=33)\end{array}$ & $\begin{array}{c}\text { AfD } \\
(\mathrm{n}=36)\end{array}$ \\
\hline $\begin{array}{l}\text { Eher „postfaktische } \\
\text { Bürger“ }\end{array}$ & 40,0 & 41,9 & 48,1 & 67,3 & 45,5 & 88,9 \\
$\begin{array}{l}\text { Eher „faktische Bürger“ } \\
\begin{array}{l}\text { Eher „postfaktische } \\
\text { Akteure“ }\end{array}\end{array}$ & 60,0 & 58,1 & 51,9 & 32,7 & 54,5 & 11,1 \\
\begin{tabular}{l} 
Eher „faktische Akteure“ \\
\hline
\end{tabular} & 96,0 & 1,1 & 3,6 & 7,3 & 2,8 & 10,0 \\
\hline
\end{tabular}

\subsection{Journalistische Reaktionen auf postfaktische Politikerinnen}

Bei möglichen Lügen und so genanntem Bullshit stehen Journalistinnen vor dem Problem, dass Lügen oder Bullshit - außer bei Geständnissen - Zuschreibungen und damit Vermutungen bleiben. Journalistinnen können also z. B. in Kommentaren nur mutmaßen bzw. in Berichten andere Politikerinnen mit entsprechenden Einschätzungen zu Wort kommen lassen. Wie reagieren Journalistinnen in einer solchen Situation? Hierzu wurden mögliche Reaktionen zu zurückliegenden Erfahrungen abgefragt. ${ }^{8}$ Journalistinnen können solch einen Verdacht explizit als mögliche Lüge bzw. Täuschungsversuch kritisieren, es als „Teil des Spiels“ thematisieren oder auf eine Thematisierung völlig verzichten.

Hier zeigt sich deskriptiv ein ambivalentes Bild (Tabelle 5). Jeweils rund 40 Prozent der Journalistinnen kritisierten ein solches vermutetes Verhalten und betrachteten es als „Teil des Spiels“, nur 3 \% thematisierten so etwas grundsätzlich nicht. In der Hypothese 3.1 wird erwartet, dass Journalistinnen, die als „postfaktische Bürgerinnen“ weniger an eine Einhaltung der Wahrheitsnorm glauben, den möglichen Verstoß gegen die Wahrheitsnorm eher kritisieren. Obwohl die deskriptive Auswertung diesen Zusammenhang vermuten lässt, ist der $\chi^{2}$-Test mit einem Cramers V $\left(\chi^{2}=0,090\right.$; Cramers V $=0,024 ; \mathrm{p}=$ $0,764)$ nicht signifikant.

In der Hypothese 3.2 ist hingegen vermutet worden, dass Journalistinnen, die als „faktische Akteurinnen“ u. a. Lügen und Bullshit als illegitim bewerten, Lügen- und Täuschungsversuche eher kritisierten. Auch diese Hypothese muss verworfen werden, $\mathrm{da}$ das Ergebnis nicht signifikant ist $\left(\chi^{2}=0,527\right.$; Cramers $\left.\mathrm{V}=0,055 ; \mathrm{p}=0,468\right)$.

Auch wenn die deskriptive Darstellung die Erwartungen der Hypothesen zu bestätigen scheint, können beide Hypothesen nicht angenommen werden. Die Annahme einer weit verbreiteten postfaktischen Politik, mit der in der Literatur vielfach eine wahrgenommene Bedrohung für den Journalismus verknüpft wird, führt offenbar nicht dazu, dass ein konkret unterstelltes postfaktisches Gebaren eher kritisiert würde. Dies lässt die Interpretation zu, dass Journalistinnen einen Lügenverdacht thematisieren, unabhängig davon, für wie verbreitet sie postfaktische Politik halten und wie sie deren Legitimität bewerten.

8 „Mitunter kann es vorkommen, dass man in einem Interview oder auf einer politischen Veranstaltung mit einer Politikerin oder einem Politiker nicht sicher ist, ob sie bzw. er die Unwahrheit sagt. Wie sind Sie mit solchen Situationen in Ihrer Berichterstattung umgegangen?"“; Antwortmöglichkeiten sind in Tabelle 5 aufgeführt. 
In Verbindung mit dem Befund, dass jeweils etwa 40 Prozent der Journalistinnen eine mögliche Täuschung kritisieren oder aber ohne Kritik darüber berichten, zeigt dies auch noch einmal die Zerrissenheit in Teilen des Journalismus: Soll man mögliche Lügen offen als solche kritisieren und damit für die Wahrheitsnorm eintreten, oder soll man das Urteil nicht eher den Leserinnen überlassen?

Tabelle 5: Reaktionen von Journalistinnen im Falle eines Lügenverdachts (Angaben in \%)

\begin{tabular}{|c|c|c|c|c|c|}
\hline & $\begin{array}{l}\text { Journalis- } \\
\text { tinnen, die } \\
\text { eher } \\
\text { „Bürgerin“ } \\
\text { einer } \\
\text { postfaktischen } \\
\text { Politik sind } \\
(n=69)\end{array}$ & $\begin{array}{l}\text { Journalis- } \\
\text { tinnen, die } \\
\text { eher } \\
\text { „Bürgerin“ } \\
\text { einer } \\
\text { faktischen } \\
\text { Politik sind } \\
\text { (n=87) }\end{array}$ & $\begin{array}{l}\text { Journalis- } \\
\text { tinnen, die } \\
\text { eher } \\
\text { "postfaktische } \\
\text { Akteurin“ } \\
\text { sind } \\
(\mathrm{n}=12)\end{array}$ & $\begin{array}{l}\text { Journalis- } \\
\text { tinnen, die } \\
\text { eher } \\
\text { „faktische } \\
\text { Akteurin“ } \\
\text { sind } \\
(\mathrm{n}=161)\end{array}$ & $\begin{array}{c}\text { Alle } \\
\text { Journalis- } \\
\text { tinnen } \\
(\mathrm{n}=194)\end{array}$ \\
\hline $\begin{array}{l}\text { Kritisieren } \\
\text { („Ich finde das } \\
\text { nicht in } \\
\text { Ordnung und } \\
\text { habe die } \\
\text { mögliche Lüge } \\
\text { bzw. den } \\
\text { Täuschungs- } \\
\text { versuch im } \\
\text { Beitrag als solche } \\
\text { kritisiert.“) }\end{array}$ & 44,9 & 42,5 & 33,3 & 44,1 & 43,3 \\
\hline $\begin{array}{l}\text { Thematisierung } \\
\text { als Spiel } \\
\text { („Das ist Teil des } \\
\text { Spiels. Ich } \\
\text { thematisiere } \\
\text { dieses Austesten } \\
\text { von Grenzen in } \\
\text { Beiträgen } \\
\text { explizit, aber } \\
\text { nicht als } \\
\text { Kritik.“) }\end{array}$ & 43,5 & 42,5 & 66,7 & 40,4 & 40,7 \\
\hline $\begin{array}{l}\text { keine } \\
\text { Thematisierung } \\
\text { („So etwas } \\
\text { thematisiere ich } \\
\text { grundsätzlich } \\
\text { nicht.“) }\end{array}$ & 1,4 & 4,6 & - & 3,7 & 3,1 \\
\hline $\begin{array}{l}\text { keine } \\
\text { Erfahrung } \\
\text { („Das ist mir } \\
\text { noch nicht } \\
\text { passiert.“) }\end{array}$ & 10,1 & 10,3 & - & 11,8 & 12,9 \\
\hline
\end{tabular}




\section{Fazit}

Die Studie hat erstmals die Verbreitung postfaktischer Annahmen bei deutschen Politikern, ihren Pressesprechern und Politikjournalisten untersucht. Bei der Frage nach der Verbreitung verschiedener Praktiken haben sich die Hypothesen bestätigt, dass Bullshit als deutlich verbreiteter wahrgenommen und als legitimeres Mittel bewertet wird als die Lüge. Hier zeigt sich, dass die bewusste Täuschung als kritikwürdiger gilt als ein gleichgültiges Verhältnis gegenüber der Wahrheit, obwohl Letzteres, so die Vermutung von Hopkin \& Rodamond (2018), gefährlicher für eine Demokratie sei. Diese Ambivalenz zwischen Verbreitung und der Legitimität postfaktischer Praktiken setzt sich bei den beiden Konstrukten, den „Bürgern“ und „Akteuren“ fort. Etwas mehr als die Hälfte der Befragten sieht sich als „Bürger“ einer postfaktischen Politik, die annehmen, dass kaum mehr jemand Wahrhaftigkeit unterstellt, während mehr als 90 Prozent der Befragten eine Politik erwarten, die ernsthaft, aufrichtig und mit Wahrheitsanspruch auftritt.

Hier wird deutlich, dass selbst die Abgeordneten und Pressesprecher, die eine Wahrheitskrise in der Politik wahrnehmen, diese verurteilen. Mit anderen Worten: Eine knappe Mehrheit wähnt sich als Teil einer postfaktischen Politik, eine überwältigende Mehrheit verurteilt eine solche. Entgegen der Hypothese wähnen sich mehr Politiker in einer postfaktischen Demokratie als Pressesprecher und Journalisten, das (unterstellte) Selbstbild ist also noch negativer als das Fremdbild. Bei einem näheren Blick auf die Unterschiede zwischen den einzelnen Parteien bestätigt sich zwar die Hypothese, dass AfDVertreter häufiger als „postfaktische Bürger“ bezeichnet werden können als Vertreter anderer Parteien. Die Ergebnisse zur Annahme, dass AfD-Vertreter solche Praktiken auch eher legitimieren, sind jedoch nicht signifikant. Es zeigen sich Indikatoren, wonach populistische Merkmale und vermehrt auch postfaktische Annahmen nicht nur bei rechtspopulistischen Parteien wie der AfD, sondern auch - mit deutlichen Abstrichen im linken Parteienspektrum zu finden sein könnten (s. auch Priester 2017).

Wie steht es um die Wahrheitsnorm in der deutschen Politik? Aus einer normativen Perspektive ist auch hier ein ambivalentes Fazit zu ziehen. Mehr als 90 Prozent aller Befragten verurteilen postfaktische Praktiken. Demnach ist es auch wahrscheinlicher, dass sie sich entsprechend verhalten (Lamnek et al. 2013). Diesen Befund relativiert ein zu vermutender Third-Person-Effekt, welcher genauer untersucht werden müsste: Man unterstellt vor allem anderen postfaktisches Verhalten, während man selbst solche Methoden ablehnt. So dürften die knapp 90 Prozent der AfD-Vertreter, die sich als „postfaktische Bürger" sehen, postfaktisches Verhalten vor allem bei den anderen Parteien unterstellen, während viele dieser Parteienvertreter wiederum vermutlich bei postfaktischer Politik zunächst an die AfD denken dürften. Mit Blick auf die Wahrnehmung der aktuellen Situation kommt folgende Erkenntnis hinzu: Eine knappe Mehrheit wähnt sich als „Bürger" einer postfaktischen Politik.

Wie aber reagieren Politiker und Pressesprecher, die der Konkurrenz den Einsatz illegitimer Methoden unterstellen? Untersuchungen in anderen Betrugsfeldern argumentieren spieltheoretisch (z. B. Keck \& Wagner 1990 zum Doping im Spitzensport), dass dies dazu führen könne, dass auch andere zu solchen Methoden greifen, um „Waffengleichheit" herzustellen. Und dennoch: Das überwältigende Ausmaß, mit dem Praktiken wie Lüge und Bullshit abgelehnt werden, macht trotz Einwänden, wie dem Problem sozialer Erwünschtheit, optimistisch. „Die komplette Entkopplung von Politik und Wahrheit erfolgt dementsprechend tatsächlich nur für den Moment der Kommunikation selbst. In einem politischen System, in welchem zwar die Orientierung der Politik an der Wissenschaft, nicht aber der grundsätzliche Zusammenhang der Logiken in Frage gestellt wurde, ist vorerst nicht damit zu rechnen, dass die politische Kommuni- 
kation immer postfaktischer wird. Solange ein Wechselverhältnis zwischen Politik und Wahrheit in starker Ausprägung besteht, kann die Falschaussage, ganz gleich ob postfaktisch oder als Lüge, als politisches Manöver nur funktionieren, solange sie die Ausnahme bleibt" (Haacke 2019, S. 48).

In einer solchen Situation kommt dem Journalismus eine zentrale Rolle zu. Berichtet er kritisch über mögliche Lügen bzw. Bullshit und aktualisiert damit die Wahrheitsnorm? Oder thematisiert er solche Methoden wie ein Sportreporter bzw. „HorseRace“-Journalist als „Teil des Spiels“ (Bennett 2016). Das Ergebnis von je 40 \%-Zustimmung scheint die ambivalente Stimmung im deutschen Journalismus wiederzugeben, in der darum gerungen wird, wie man mit solchen Methoden umgeht.

Weiterer Forschungsbedarf besteht nicht nur zu verschiedenen journalistischen Thematisierungsstrategien der beschriebenen Methoden. Wie wirken verschiedene Berichterstattungsstrategien? Fühlen sich Leser bevormundet bei deutlich geäußerter Kritik an Politikern? Oder wünschen sie sich eine solche kritische Berichterstattung? Mit einer Wiederholung der Studie könnte zudem gezeigt werden, ob die verbreitete Unterstellung einer postfaktischen Politik dazu führt, dass Politiker und ihre Pressesprecher Methoden wie Lüge und Bullshit legitimer bewerten - und dann ggf. auch selbst nutzen. Schließlich könnte das Konzept der politischen Kommunikationskultur in der erweiterten Perspektive zum Einsatz kommen, indem auch Bürger und deren Einstellungen berücksichtigt würden, um damit z. B. Unterschiede zwischen Bundesländern zu untersuchen.

Auch wenn in der Studie nicht danach gefragt wurde, ob ein Politiker schon einmal gelogen hat, so unterliegen auch die Legitimitätseinschätzungen in hohem Maße dem Problem der sozialen Erwünschtheit. Da die AfD sich seit vielen Jahren Vorwürfen von Lüge und Fake News ausgesetzt sieht, ist nicht auszuschließen, dass ihre Abgeordneten bzw. Pressesprecher in besonderer Weise sensibilisiert sind und hier in höherem Ausmaß sozial erwünscht geantwortet haben als andere Parteienvertreter. Die Rücklaufquote der Studie war zwar noch mit denen anderer Studien vergleichbar. Insgesamt setzt sich aber der Trend zurückgehender Rücklaufquoten bei Elitenbefragungen fort. Angesichts der intensiven Erforschung dieser und vergleichbarer Felder ist zu erwarten, dass sich diese Entwicklung fortsetzt und für die Forschung zunehmend zu Problemen führt.

\section{Literatur}

Arendt, F., Haim, M., \& Beck, J. (2019). Fake News, Warnhinweise und perzipierter Wahrheitsgehalt: Zur unterschiedlichen Anfälligkeit für Falschmeldungen in Abhängigkeit von der politischen Orientierung. Publizistik, 64(2), 181-204.

Arendt, H. (2013). Wabrheit und Lüge in der Politik: zwei Essays. München: Piper.

Arora, M. (2020). Post-truth and marketing communication in technological age. In Handbook of Research on Innovations in Technology and Marketing for the Connected Consumer (S. 94-108). Hershey: IGI Global.

Batinic, B., \& Moser, K. (2005). Determinanten der Rücklaufquote in Online-Panels. Zeitschrift für Medienpsychologie, 17(2), 64-74.

Baudrillard, J. (1978a). Agonie des Realen. Berlin: Merve Verlag.

Baudrillard, J. (1978b). Kool Killer oder der Aufstand der Zeichen. Berlin: Merve Verlag.

Beck, U. (1986). Risikogesellschaft. Frankfurt: Suhrkamp.

Bennett, W. L. (2016). News: The politics of illusion (10. Aufl.). Chicago: University of Chicago Press.

Bentele, G., \& Seidenglanz, R. (2004). Das Image der Image-Konstrukteure. Eine repräsentative Studie zum Image der PR-Branche in Deutschland und eine Journalistenbefragung. Leipzig: Universität Leipzig.

Best, H., Edinger, M., Gerstenhauer, D., \& Vogel, L. (2010). Jenaer Parlamentarierbefragung 2010. Ausgewählte Ergebnisse. Jena: Friedrich-Schiller-Universität Jena. 
Blumler, J. G., \& Gurevitch, M. (1995). The crisis of public communication. London: Routledge.

Boberg, S., Schatto-Eckrodt, T., Frischlich, L., \& Quandt, T. (2018). The moral gatekeeper? Moderation and deletion of user-generated content in a leading news forum. Media and Communication, 6(4), 58-69.

Carlson, M. (2018). The information politics of journalism in a post-truth age. Journalism Studies, 19(13), 1879-1888.

Citrin, J., \& Stoker, L. (2018). Political trust in a cynical age. Annual Review of Political Science, $21,49-70$.

Corner, J. (2017). Fake news, post-truth and media-political change. Media, Culture E Society, 39(7), 1100-1107.

d'Ancona, M. (2017). Post-truth: The new war on truth and how to fight back. London: Ebury Press.

Eldridge, S. A., \& Bødker, H. (2018). Negotiating uncertain claims: Journalism as an inferential community. Journalism Studies, 19(13), 1912-1922.

Engesser, S., Fawzi, N., \& Larsson, A. O. (2017). Populist online communication: Introduction to the special issue. Information, Communication E Society, 20(9), 1279-1292.

Enli, G., \& Rosenberg, L. T. (2018). Trust in the age of social media: Populist politicians seem more authentic. Social Media + Society, 4(1), 1-11.

Frankfurt, H. G. (2006). Bullshit. Frankfurt am Main: Suhrkamp.

Fraune, C., \& Knodt, M. (2018). Sustainable energy transformations in an age of populism, posttruth politics, and local resistance. Energy Research E Social Science, 43, 1-7.

Gibson, T. (2018). The Post-truth double-helix: Reflexivity and mistrust in local politics. International Journal of Communication, 12, 19, 3167-3185, https://ijoc.org/index.php/ijoc/article/ view/7738.

Giddens, A. (1995). Konsequenzen der Moderne. Frankfurt am Main: Suhrkamp.

Gil de Zúñiga, H., Koc Michalska, K., \& Römmele, A. (2020). Populism in the era of Twitter: How social media contextualized new insights into an old phenomenon. new media E society, 22(4), 585-594.

Godler, Y. (2020). Post-post-truth: An adaptationist theory of journalistic verism. Communication Theory, 30(2), 169-187.

Gugushvili, A., Koltai, J., Stuckler, D., \& McKee, M. (2020). Votes, populism, and pandemics. International Journal of Public Health, 65(6), 721-722.

Haacke, N. (2019). Kontingente Kopplung. Postfaktische Kommunikation im Spannungsfeld von Politik und Wissenschaft. Soziologiemagazin, 12(2), 33-50.

Hallahan, K., Holtzhausen, D., van Ruler, B., Verč́č, D., \& Sriramesh, K. (2007). Defining strategic communication. International Journal of Strategic Communication, 1(1), 3-35.

Hameleers, M., \& Minihold, S. (2020). Constructing discourses on (un)truthfulness: Attributions of reality, misinformation, and disinformation by politicians in a comparative social media setting. Communication Research. https://doi.org/10.1177/0093650220982762.

Harsin, J. (2018). Post-truth and critical communication studies. In Oxford Research Encyclopedia of Communication. https://doi.org/10.1093/acrefore/9780190228613.013.757.

Higgins, K. (2016). Post-truth: a guide for the perplexed. Nature, 540(7631), 9.

Hölig, S. \& Hasebrink, U. (2020). Reuters Institute Digital News Report 2020 - Ergebnisse für Deutschland. Hamburg: Verlag Hans-Bredow-Institut.

Hoffjann, O. (2013). Die Wirklichkeit der Public Relations. Medien E Kommunikationswissenschaft $M E K, 61(1), 76-90$.

Hoffjann, O. (2020). The politician as a player: political strategic communication as a play. Journal of Communication Inquiry, https://doi.org/10.1177/0196859920977123.

Hoffjann, O. (2021). Politische Kommunikation im Spielmodus. In S. Schicha, I. Stapf, \& S. Sell (Hrsg.), Medien und Wabrheit. Medienethische Perspektiven auf Desinformation, Lügen und „Fake News" (S. 205-222). Baden-Baden: Nomos.

Hoffjann, O., \& Lohse, M. (2016). „Berliner Käseglocke“ versus „Hannoveraner Verhältnisse “? Eine vergleichende Untersuchung der Beziehungen von Politik und Journalismus auf Bundesund Länderebene. Medien E Kommunikationswissenschaft MEK, 64(2), 193-218. 
Hopkin, J., \& Rosamond, B. (2018). Post-truth politics, bullshit and bad ideas: 'Deficit fetishism' in the UK. New political economy, 23(6), 641-655.

Ihlen, Ø., Gregory, A., Luoma-aho, V., \& Buhmann, A. (2019). Post-truth and public relations: Special section introduction. Public Relations Review, 45(4), 101844, https:/doi.org/10.1016/ j.pubrev.2019.101844.

Kaldewey, D. (2013). Wabrheit und Nützlichkeit: Selbstbeschreibungen der Wissenschaft zwischen Autonomie und gesellschaftlicher Relevanz. Bielefeld: transcript.

Kalpokas, I. (2019). Post-Truth and the Changing Information Environment. In P. Baines, N. O'Shaughnessy, \& N. Snow (Hrsg.), The SAGE Handbook of Propaganda (S. 71-84). SAGE Publications Limited.

Keck, O., \& Wagner, G. (1990). Asymmetrische Information als Ursache von Doping im Hochleistungssport. Eine Analyse auf Basis der Spieltheorie. Zeitschrift für Soziologie, 19(2),108-116.

King, M. F., \& Bruner, G. C. (2000). Social desirability bias: A neglected aspect of validity testing. Psychology \& Marketing, 17(2), 79-103.

Kohring, M. (2004). Vertrauen in Journalismus. Theorie und Empirie. Konstanz: UVK.

Lamnek, S., Olbrich, G., \& Schäfer, W. J. (2013). Tatort Sozialstaat: Schwarzarbeit, Leistungsmissbrauch, Steuerhinterziehung und ibre (Hinter) Gründe. Opladen: Leske + Buderich.

Lewandowsky, M., Giebler, H., \& Wagner, A. (2016). Rechtspopulismus in Deutschland. Eine empirische Einordnung der Parteien zur Bundestagswahl 2013 unter besonderer Berücksichtigung der AfD. Politische Vierteljabresschrift, 57(2), 247-275.

Lewandowsky, S., Ecker, U. K., \& Cook, J. (2017). Beyond misinformation: Understanding and coping with the "post-truth" era. Journal of applied research in memory and cognition, 6(4), 353-369.

Luhmann, N. (1991). Die Politik der Gesellschaft. Frankfurt am Main: Suhrkamp.

Luhmann, N. (1993). Erleben und Handeln. In Soziologische Aufklärung 3. Soziales System, Gesellschaft, Organisation (S. 67-80). Opladen: Westdeutscher Verlag.

Luhmann, N. (1994). Die Wissenschaft der Gesellschaft. Frankfurt am Main: Suhrkamp.

MacKenzie, A., \& Bhatt, I. (2020). Opposing the power of lies, bullshit and fake news: the value of truth. Postdigital Science and Education, 2(1), 217-232.

Mair, J. (2017). Post-truth anthropology. Anthropology Today, 33(3), 3-4.

Maurer, M., \& Jost, P. (2020). Das Ende der Debattenkultur? Wie die AfD Negativität und Inzivilität in den Bundestag trägt und wie die anderen Parteien darauf reagieren. Zeitschrift für Politik, 67(4), 473-490.

McDevitt, M., \& Ferrucci, P. (2018). Populism, journalism, and the limits of reflexivity: The case of Donald J. Trump. Journalism Studies, 19(4), 512-526.

McIntyre, L. (2018). Post-truth. Cambridge: MIT Press.

McLeod, J. M. \& Chaffee, S. H. (1972): The construction of social reality. In J. T. Tedeschi (Hrsg.), The social influence processes (S. 50-99). Chicago: Aldine,

Meibauer, J. (2013). Bullshit als pragmatische Kategorie. Linguistische Berichte, 2013(235), 267-292.

Mitterer, J. (1992). Das Jenseits der Philosophie. Wider das dualistische Erkenntnisprinzip. Wien: Passagen.

Mitterer, J. (2001). Die Flucht aus der Beliebigkeit. Frankfurt am Main: S. Fischer.

Muiller, J. W. (2016). What Is Populism? Pennsylvania: University of Pennsylvania Press.

Nuernbergk, C., \& Schmidt, J. H. (2020). Twitter im Politikjournalismus. Publizistik, 65(1), 41-61.

Ohliger, U. A., \& Vögele, C. (2020). Objektive Informationsquelle oder Mittel zur politischen Instrumentalisierung? Wie Landtagskorrespondenten die Bedeutung und Effekte der medialen Umfrageberichterstattung wahrnehmen. Medien $\mathcal{E}$ Kommunikationswissenschaft $M \mathcal{E} K, 68(1-$ 2), 113-141.

Pappas, T. S. (2016). Modern populism: Research advances, conceptual and methodological pitfalls, and the minimal definition. In Oxford Research Encyclopedia of Politics. https://doi.org/ 10.1093/acrefore/9780190228637.013.17.

Pfetsch, B. (2003). Politische Kommunikationskultur. Politische Sprecher und Journalisten in der Bundesrepublik und den USA im Vergleich. Wiesbaden: VS Verlag. 
Pfetsch, B., \& Mayerhöffer, E. (2011). Vordergründige Nähe. Zur Kommunikationskultur von Politik- und Medieneliten in Deutschland. Medien E Kommunikationswissenschaft MEK, 59(1), 40-59.

Pörksen, B. (2018). Die große Gereiztheit: Wege aus der kollektiven Erregung. München: Carl Hanser Verlag.

Pontzen, D. (2013). Politiker in der Medialisierungsspirale? Eine Abgeordneten-Befragung auf Landes-, Bundes- und EU-Ebene. Baden-Baden: Tectum-Verlag.

Priester, K. (2017). Linkspopulismus - die andere Seite der populistischen Medaille. Forschungsjournal Soziale Bewegungen, 30(2), 50-59.

Romano, A. (2017). Asserting journalistic autonomy in the 'post-truth' era of 'alternative facts': Lessons from reporting on the orations of a populist leader. Asia Pacific Media Educator, 27(1), 51-66.

Quandt, T., Frischlich, L., Boberg, S., \& Schatto-Eckrodt, T. (2019). Fake news. In T. P. Vos, F. Hanusch, A. Sehl, D. Dimitrakopoulou, \& M. Geertsema-Sligh (Hrsg.), The International Encyclopedia of Journalism Studies (S. 1-6). Boston: Wiley Blackwell.

Şahin, O., Johnson, R., \& Korkut, U. (2021). Policy-making by tweets: discursive governance, populism, and Trump Presidency. Contemporary Politics. https://doi.org/ 10.1080/13569775.2021.1935009.

Scholl, A. (2018). Die andere Seite: Das Misstrauen des Journalismus. In O. Hoffjann, \& R. Seidenglanz (Hrsg.), Allmächtige PR, obnmächtige PR (S. 107-133). Wiesbaden: Springer VS.

Scholl, A. (2019). Ideologiekritik und Kontingenz(erfahrung) am Beispiel Fake News: Der Beitrag des Radikalen Konstruktivismus. In U. Krüger, \& S. Sevignani (Hrsg.), Ideologie, Kritik, Öffentlichkeit. Verhandlungen des Netzwerks Kritische Kommunikationswissenschaft (S. 46-64). Leipzig: Universität Leipzig.

Schwaiger, L., \& Eisenegger, M. (2021). Die Rahmung von Wahrheit und Lüge in Online-Gegenöffentlichkeiten. Eine netzwerkanalytische Untersuchung auf Twitter. In C. Schicha, I. Stap, \& A. Sell (Hrsg.), Medien und Wabrbeit. Medienethische Perspektiven auf Desinformation, Lügen und „Fake News" (S. 359-376). Baden-Baden: Nomos.

Seidenglanz, R. (2015). Vertrauen und Public Relations. Theorieentwicklung und empirische Umsetzung. Dissertationsschrift. Leipzig: Universität Leipzig.

Spieß, E., Frieß, D., \& Schulz, A. (2020). Populismus auf Facebook. Zeitschrift für Politikwissenschaft, 30(2), 219-240.

Tandoc Jr, E. C., Jenkins, J., \& Craft, S. (2019). Fake news as a critical incident in journalism. Journalism Practice, 13(6), 673-689.

Tumber, H., \& Waisbord, S. (2021). Media, disinformation, and populism: Problems and responses. In H. Tumber, \& S. Waisbord (Hrsg.), The Routledge Companion to Media Disinformation and Populism (S. 13-25). London: Routledge.

Vogelmann, F. (2018). The Problem of Post-Truth. Rethinking the Relationship between Truth and Politics. BEHEMOTH - A Journal on Civilisation, 11(2), 18-37.

van Dyk, S. (2017). Krise der Faktizität? Über Wahrheit und Lüge in der Politik und die Aufgabe der Kritik. PROKLA. Zeitschrift für kritische Sozialwissenschaft, 47(188), 347-368.

Wagner, M. C., \& Boczkowski, P. J. (2019). The reception of fake news: The interpretations and practices that shape the consumption of perceived misinformation. Digital journalism, 7(7), 870885.

Waisbord, S. (2018). Truth is what happens to news: On journalism, fake news, and post-truth. Journalism studies, 19(13), 1866-1878.

Washington Post (2020). In 1,386 days, President Trump has made 29,508 false or misleading claims. https://www.washingtonpost.com/graphics/politics/trump-claims-database/?itid=lk_inline_ manual_4 [19.10.2021].

Weber, S. (2019). Ibiza-Affäre, Schredder-Affäre, Casinos-Affäre - und wie weiter? Telepolis am 26.08.2019, https://www.heise.de/tp/features/Ibiza-Affaere-Schredder-Affaere-Casinos-Af faere-und-wie-weiter-4504405.html?seite=all [19.10.2021].

Weischenberg, S., Malik, M., \& Scholl, A. (2006). Die Souffleure der Mediengesellschaft. Report über die Journalisten in Deutschland. Konstanz: UVK. 
M\&K 69. Jahrgang 4/2021

Zimmermann, F., \& Kohring, M. (2018). „Fake News“ als aktuelle Desinformation. Systematische Bestimmung eines heterogenen Begriffs. Medien $\mathcal{E}$ Kommunikationswissenschaft $M \mathcal{E} K, 66(4)$, 526-541.

Zmerli, S., \& van der Meer, T. W. (Eds.) (2017). Handbook on political trust. Cheltenham: Edward Elgar Publishing. 